\title{
Situation actuelle de la mécanique quantique: des difficultés conceptuelles subsistent"
}

\author{
Franck Laloë
}

Laboratoire Kastler Brossel, ENS-UMPC, 75005 Paris, France

\section{Résumé}

La mécanique quantique est l'une des plus grandes réussites de toute l'histoire des sciences. Ses prédictions ont été vérifiées dans un très grand nombre de cas, avec parfois une précision fantastique de $10^{-12}$. Historiquement, elle s'est développée en plusieurs étapes distinctes, chacune d'entre elles impliquant des interprétations différentes, parfois opposées. Puis s'est dégagée une version dite standard, dans la ligne des travaux de von Neumann et de Dirac, qui est généralement celle présentée dans les ouvrages et enseignée. Mais de réelles difficultés conceptuelles subsistent; nous sommes loin d'un consensus universel sur la meilleure façon d'interpréter la nature de la fonction d'onde, la mesure, etc. et leur relation (éventuelle) avec la « réalité physique ». C'est pourquoi de nombreuses présentations et/ou interprétations de la mécanique quantique ont été proposées. L'exposé en décrira quelquesunes : bien sûr celle dite de Copenhague (Bohr), l'interprétation statistique (à laquelle on attache souvent le nom d'Einstein), etc. avec également les trois interprétations non-standard les plus célèbres : $\mathrm{dBB}$ (de Broglie-Bohm), GRW/CSL (équation de Schrödinger stochastique), Everett (parfois dite «des mondes multiples »)

(*) Ce chapitre est issu du livre Comprenons nous vraiment la mécanique quantique, Franck Laloe, EDP Sciences/CNRS Editions, 2011. 


\section{Abstract}

\section{Current situation of quantum mechanics: some conceptual problems remain}

Quantum mechanics is one of the most successful theories of all physics. Its predictions have been verified in a huge number of situations, sometimes with a fantastic accuracy of $10^{-12}$. Historically, it was developed in several stages, each of them implying a different interpretation, sometimes opposite of the others. A "standard" version then emerged, in the line of the contributions of von Neumann and Dirac, which is the version generally found in textbooks. Nevertheless, real conceptual difficulties remain: we are far from a universal consensus concerning the best way to interpret the nature of the wave function, the measurement process, etc. and their possible relation with "physical reality". This is the reason why many interpretations of quantum mechanics have been proposed. A few will be described during the talk: the Copenhagen interpretation (Bohr), the statistical interpretation (Einstein, Ballentine, etc.) as well as the three most famous nonstandard interpretations: dBB (de Broglie Bohm), GRWICSL (Stochastic Schrödinger dynamics) and Everett (sometimes described as "many worlds").

Un certain nombre des difficultés conceptuelles de la mécanique quantique, déjà identifiées par ses inventeurs, subsistent à l'heure actuelle. Que le lecteur n'en retire pas l'impression qu'il s'agit d'une théorie peu solide! L'arbre ne doit pas cacher la forêt : indépendamment de ces difficultés très réelles, la mécanique quantique est assurément l'une des théories les plus couronnées de succès de toute la science. On peut même considérer que sa faculté d'adaptation aux situations les plus variées est sa caractéristique la plus extraordinaire ; elle continue à être toujours aussi efficace dans ses prédictions en accompagnant les progrès expérimentaux qui permettent constamment d'avoir accès à de nouvelles situations physiques que les pères fondateurs de la théorie n'avaient aucun moyen de prévoir. De fait, il existe très peu de théories dont la vérification ait été faite avec la même précision, et ceci dans des domaines d'application variés. Il subsiste cependant que, sur le plan conceptuel, des difficultés subsistent, et ce sont elles qui font l'objet du présent chapitre. Comme nous le verrons, la plupart mettent en jeu le processus de mesure quantique et la nature du phénomène aléatoire qui se produit à cette occasion.

Dans le point de vue de Von Neumann ce n'est que lorsqu'une mesure est effectuée que des sauts aléatoires se produisent, selon le postulat de réduction du vecteur d'état. Sinon, en général la fonction d'onde évolue progressivement, d'une façon parfaitement prévisible et continue, selon l'équation de Schrödinger. Bien évidemment, utiliser deux postulats aussi différents pour l'évolution du même objet mathématique' est une situation tout à fait

(I) Au lieu du «point de vue de Schrödinger », il est équivalent d'utiliser « point de vue de Heisenberg »; ; 'évolution continue du vecteur d'état est alors transférée vers les observables du système (opérateurs agissant sur son espace des états), tandis que le vecteur d'état reste indépendant du temps en l'absence de mesure. Les observables évoluent de façon continue, que des mesures soient effectuées ou pas. Mais le vecteur d'état doit encore obéir à des règles différentes suivant la situation: la plupart du temps il reste parfaitement constant, mais lors d'une mesure il saute brusquement vers une nouvelle valeur prenant en compte l'information nouvelle. L'acquisition d'information est donc toujours considérée comme un processus physique totalement différent de l'évolution « normale », ce qui introduit la nécessité de bien définir la frontière entre les deux processus. 
inhabituelle en physique. Cette idée était d'ailleurs une totale nouveauté lorsqu'elle a été introduite, et reste actuellement unique dans toute la physique, ainsi qu'une source de difficultés - en particulier celles liées à la compatibilité entre les deux postulats. II serait plus naturel de voir les processus de mesure, non pas comme complètement à part, mais simplement comme un processus d'interaction ordinaire entre un système mesuré (qui peut éventuellement être microscopique) et un appareil de mesure macroscopique ; il semblerait bien préférable d'utiliser une théorie plus générale où un tel processus soit traité dans le cadre des lois ordinaires de la physique, de sorte que la distinction entre évolution ordinaire et mesure devienne inutile. En d'autres termes, pourquoi donc attribuer aux mesures un caractère aussi particulier, de sorte que deux postulats distincts deviennent nécessaires ? Où exactement s'arrête le domaine d'application du premier pour laisser la place au second? Plus précisément, parmi toutes les interactions - ou perturbations - que peut subir un système physique, lesquelles doivent être considérées comme normales (évolution de Schrödinger), et lesquelles comme une mesure (réduction du vecteur d'état) ? On voudrait mieux comprendre la raison pour laquelle la physique nous demande d'introduire une frontière entre deux mondes et sa nature : le monde de Schrödinger, parfaitement continu et déterministe, et celui très différent de Born et de la mesure, où l'évolution déterministe cesse pour laisser la place à des processus discontinus et aléatoires.

Dans le point de vue de Bohr également, les processus de mesure et les appareils associés sont clairement « discriminés » du système étudié. Dans l'univers de Bohr, l'évolution se fait généralement de façon libre et régulière selon l'équation de Schrödinger. Mais, dans le cas particulier des événements mettant en jeu un transfert d'information vers des observateurs, s'introduit une composante aléatoire fondamentale. De plus, ces opérations de mesure sont en quelque sorte considérées comme des «bulles fermées » incluses dans cette évolution générale, des événements clos de leur début à leur fin et s'étendant sur une région d'espace-temps, et qu'il ne faut pas chercher à décomposer de façon plus fine en événements relativistes. Ces processus sont caractérisés par le fait qu'une intelligence humaine pose une question à la Nature, dont résulte une réponse unique, mais non-déterministe.

Que nous préférions le point de vue de Bohr ou de Von Neumann, nous sommes en face d'un problème logique qui n'existait pas en mécanique classique, lorsque personne ne pensait que les mesures donnant de l'information doivent être traitées à part parmi les processus physiques. Bohr nous apprend que nous ne devrions pas tenter de transposer notre expérience journalière du monde vers des systèmes microscopiques ; certes mais alors, pour chaque expérience, où mettre exactement la limite entre les deux mondes ? Peut-on réellement se contenter de remarquer que la distance entre macroscopique et microscopique est tellement grande que la position de la frontière entre ces deux mondes est sans importance?

On peut de plus trouver très surprenant qu'en physique moderne il faille faire jouer un rôle aussi spécial à un " observateur » ou à l'information acquise, ce qui donne à la théorie une coloration anthropocentrique inattendue, comme dans l'astronomie médiévale. Devons-nous réellement rejeter comme non-scientifique l'idée d'une description scientifique de systèmes physiques isolés, simplement parce que nous ne les observons pas ? Si les observateurs sont si importants, comment doit-on les définir exactement? 
Par exemple, est-ce qu'un animal peut réaliser une expérience et réduire le vecteur d'état, ou est-ce l'unique apanage des êtres humains ? Bell avec humour a posé la question [2] : « La fonction d'onde de l'Univers était-elle en train d'attendre d'effectuer son premier saut pendant des milliers de millions d'années jusqu'à l'apparition de la première créature vivante unicellulaire? Ou a-t-il fallu attendre un peu plus l'apparition d'un observateur plus qualifié - avec un doctorat d'état ? »-voir aussi la référence [3], en particulier son titre. Ces questions très générales sont difficiles et quelque peu philosophiques, par exemple London et Bauer [4] ont proposé que la capacité de jouer le rôle d'un observateur en théorie quantique soit liée à la faculté d'introspection d'une créature vivante.

Une autre difficulté provient du caractère aléatoire des prédictions de la mécanique quantique. En soi, une telle situation est habituelle en physique, où par exemple la mécanique statistique classique fait un usage constant de probabilités. Mais les probabilités sont alors associées étroitement à la notion d'ensemble statistique : on suppose par exemple que le système est choisi au hasard parmi un très grand nombre de systèmes similaires définissant un même ensemble. Certes, tous ces systèmes partagent un certain nombre de propriétés contenues dans la description statistique, mais une description plus précise peut révéler qu'à un niveau plus fin, ils diffèrent aussi par un certain nombre de spécificités, ou par des influences minimes qu'ils ont subies, etc. En d'autres termes, chaque système individuel possède plus de propriétés physiques que celles spécifiées pour l'ensemble. La même question se transpose alors immédiatement à la mécanique quantique : si ses prédictions sont des probabilités, faut-il en conclure qu'elle ne décrit que des ensembles de systèmes ? Ou faut-il au contraire considérer que le vecteur d'état fournit la description la plus précise possible d'un seul système physique?

Il est impossible de résumer tout ce qui a été écrit sur ces difficultés, en particulier sur le rôle exact et l'évolution du vecteur d'état, en un nombre raisonnable de pages. A titre d'illustration, nous discuterons quelques exemples, la chaîne sans fin de Von Neumann $(\S \mathrm{I})$, le chat de Schrödinger ( $(2)$, l’ami de Wigner ( $(3)$ et les mesures négatives ( $\$ 4)$. Puis (§5) nous proposerons un certain nombre de citations anciennes ou plus récentes, qui permettent de se faire une bonne idée de la variété des positions possibles, avant de terminer par un paragraphe mentionnant quelques points qui ont donné lieu à des erreurs dans le passé (\$6).

\section{La chaîne (ou régression) sans fin de Von Neumann}

Von Neumann, dans son traité publié en 1932 (chapitres 4-6 de [5]), a introduit une théorie explicite de la mesure quantique. À la différence du point de vue de Bohr, Von Neumann considère l'appareil de mesure comme un système quantique sur le même plan que le système mesuré. II étudie ce qui se produit lorsque le système mesuré $S$ est mis en contact avec un appareil de mesure $M$ et interagit avec lui pendant un certain temps. $M$ inclut un « pointeur » (ou aiguille de cadran) macroscopique qui, une fois que l'interaction est terminée, a atteint une position qui dépend de l'état initial de S. Nous donnons ci-des- 
sous une introduction aux idées générales concernant la chaîne de Von Neumann, sans écrire d'équations.

Commençons par un cas particulier : celui où, initialement, le système $S$ était dans un état propre de l'observable mesurée $A$ caractérisé par une de ses valeurs propres ${ }^{2} a$. La position atteinte par le pointeur dépend alors de l'état propre ; on peut donc dire que l'observation de la position macroscopique de $\mathrm{P}$ revient à mesurer l'observable et obtenir le résultat $a$.

Mais, de façon plus générale, initialement $S$ n'est pas dans un état propre de l'observable mesurée $A$, mais dans une superposition de tels états. Comme l'équation de Schrödinger est linéaire, elle prédit que le système total $\mathrm{S}+\mathrm{M}$ atteint après interaction une superposition d'états. Dans les différentes composantes de cette superposition, le pointeur se trouve dans des positions différentes, chacune correspondant à un résultat de mesure différent ; on dit alors que les deux systèmes $S$ et $M$ sont dans un état d'intrication quantique. Ainsi, la linéarité de l'équation de Schrödinger la rend incapable d'effectuer une sélection entre ces résultats ; elle ne peut conduire qu'à une superposition de tous les résultats, comme si aucun résultat bien défini n’avait émergé de la mesure!

Pour résoudre ce problème, on pourrait alors être tenté d'ajouter un « second étage » au processus, et d'introduire un second appareil de mesure M' dont le rôle sera de déterminer la position du pointeur de $\mathrm{M}$. Mais le processus se répète et la linéarité de l'équation de Schrödinger conduit à une superposition plus étrange encore puisque, cette fois, elle contient $\mathrm{S}+\mathrm{M}+\mathrm{M}^{\prime}$ ainsi que des positions différentes des pointeurs de $\mathrm{M}$ et $\mathrm{M}$ '. Par

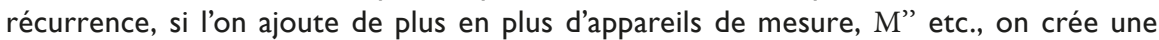
chaine de plus en plus longue de systèmes corrélés, sans jamais arriver à sélectionner un seul résultat de mesure. Ce processus récurrent $M, M^{\prime}, M^{\prime \prime}, \ldots$ est appelé régression de Von Neumann, ou encore chaîne infinie de Von Neumann. On peut résumer toute cette discussion par une seule phrase : «L'unicité des résultats ne peut pas émerger de la seule équation de Schrödinger ; elle crée en effet une chaîne de superpositions cohérentes qui se propage sans fin $»$.

Un exemple simple est utile pour illustrer le processus de façon plus concrète. Supposons par exemple que nous considérions un atome de spin $1 / 2$ qui pénètre dans le champ magnétique d'un analyseur de Stern et Gerlach. Si l'état initial du spin correspond à une direction qui est, soit parallèle, soit antiparallèle à la direction d'analyse de l'appareil (définie par la direction de son champ magnétique), le spin est initialement dans un état propre correspondant à la mesure; le paquet d'ondes est dévié vers le haut dans le premier cas, vers le bas dans le second. Mais si la direction initiale du spin était perpendiculaire à la direction d'analyse, la fonction d'onde se sépare en deux paquets d'ondes différents, l'un dévié vers le haut, l'autre vers le bas ; c'est à nouveau une conséquence directe de la linéarité de l'équation de Schrödinger. Lorsqu'ils se propagent plus loin, chacun des deux paquets d'onde peut frapper un détecteur avec lequel il interagit et dont il modifie l'état (ainsi que le sien) ; par exemple, les atomes de spin I/2 sont ionisés et produisent des électrons, de sorte que la superposition linéaire englobe maintenant plus de particules. De plus, lorsqu'une cascade d'électrons est produite dans un multiplicateur d'électrons,

(2) Pour simplifier, nous supposons que cette valeur propre n'est pas dégénérée, mais la généralisation à des valeurs propres dégénérées est possible. 
tous ces électrons supplémentaires deviennent également partie de la superposition. II n'y a pas de limite intrinsèque à ce qui devient bientôt la propagation d'une chaîne sans fin : rapidement, la linéarité de l'équation de Schrödinger conduit à un vecteur d'état qui est la superposition cohérente d'états où un nombre macroscopique de particules, des courants macroscopiques et, pourquoi pas, des aiguilles d'appareils ou des enregistreurs qui écrivent des chiffres macroscopiques sur une feuille de papier! Si nous restons dans le cadre de l'équation de Schrödinger, rien ne nous permet de stopper la progression de cette régression infinie. Devons-nous alors accepter l'idée qu'à la fin c'est le cerveau de l'expérimentateur, lorsqu'il prend connaissance des résultats imprimés, et donc un être humain doué de conscience, qui entre dans une telle superposition?

La notion même de cerveau ou de conscience se trouvant dans une superposition macroscopique n'a rien d'intuitif ni de très clair ; personne n'a jamais observé à la fois deux résultats contradictoires. Est-ce que cette situation étrange devrait être vue comme celle où un résultat expérimental imprimé sur une feuille de papier ressemblerait à deux diapositives superposées, ou une photographie doublement exposée ? En pratique, nous savons bien que nous observons toujours un résultat unique et bien défini dans une expérience ; il semble donc que la superposition linéaire se soit réduite d'une façon ou d'une autre avant de nous atteindre - probablement avant même qu'elle ne devienne suffisamment macroscopique pour mettre en jeu des appareils de mesure. II semble alors évident ${ }^{3}$ qu'une théorie raisonnable devrait briser quelque part la chaîne de Von Neumann, quand (ou peut-être avant) elle atteint le monde macroscopique. Cette opération porte souvent le nom de " coupure de Heisenberg » (« Heisenberg cut » en anglais). Mais quand et où exactement effectuer cette coupure?

Von Neumann conclut à partir de son analyse que, effectivement, il n'est pas possible de formuler les lois de la mécanique quantique sans faire référence à la prise de conscience humaine. II considère l'émergence d'un résultat unique lors d'une mesure comme un élément irréductible de la théorie. La solution du problème demande alors l'introduction d'un postulat spécial, le « postulat de projection du vecteur d'état » qui force l'émergence de ce résultat unique dès qu'un observateur prend connaissance du résultat. La notion de régression de Von Neumann est également à la source du phénomène de décohérence. Le mot « décohérence » est en fait plutôt utilisé habituellement pour désigner le tout début de la chaîne, au moment où le nombre de degrés de liberté mis en jeu reste encore relativement limité. Mais la notion générale de chaîne de Von Neumann inclut tous les stades de la régression, ce stade initial comme sa prolongation, qui se propage jusqu'à l'autre extrême où le paradoxe devient inacceptable parce que trop contraire au bon sens : le chat de Schrödinger.

\section{Le chat de Schrödinger}

La fameuse histoire du chat de Schrödinger (1935) illustre le même problème, mais d'une façon différente.

(3) Peut-être pas si évident après tout? De fait, il existe une interprétation de la mécanique quantique qui repose précisément sur l'idée que la chaîne n'est jamais rompue : l'interprétation d'Everett. 


\section{I L'argument}

Le chat n'apparaît que dans quelques lignes dans le contexte d'une discussion bien plus générale dans un article de Schrödinger [7] dont le titre est « La situation actuelle de la mécanique quantique $॥$. II écrit en allemand (pour une traduction en anglais, voir [8]) On peut même imaginer des situations tout à fait grotesques. Un chat est enfermé dans une boîte en acier, dans laquelle se trouve le dispositif diabolique suivant (sur lequel le chat ne peut avoir aucune action) : dans un compteur Geiger se trouve une toute petite quantité de matière radioactive, si petite qu'il faudra par exemple une heure pour avoir des probabilités égales que l'un des noyaux se désintègre, ou alors aucun. En cas de désintégration, le compteur à décharge se déclenche et, par un relais, relâche un marteau qui vient briser une petite ampoule contenant de l'acide cyanhydrique (poison violent). Si on laisse à luimême l'ensemble du système pendant une heure, on dirait que le chat est toujours vivant seulement si entre-temps aucun noyau ne s'est désintégré. La première désintégration atomique l'aurait empoisonné. La fonction $\Psi$ du système total exprimerait cette situation en contenant un mélange (une superposition de composantes) où le chat est vivant et mort, avec des poids égaux.

Ce type de cas est typique d'une situation où une indétermination initialement restreinte au domaine atomique se propage vers une indétermination macroscopique, qui peut alors être résolue par observation directe. II nous empêche d'accepter trop naïvement comme valable un modèle «flou » pour représenter la réalité. En lui-même, il n'a rien qui soit peut clair ou contradictoire. Il existe une différence entre une photo bougée ou floue à cause d'une mauvaise mise au point et une photo de nuages et de volutes de fumée.

En d'autres termes, Schrödinger considère une chaîne de Von Neumann, qui part d'un (ou quelques) noyau atomique, qui peut émettre un photon gamma, qui est détecté par un détecteur à rayons gamma, qui est suivi d'un étage d'amplification, qui alors déclenche un système mécanique macroscopique qui ouvre automatiquement une bouteille de poison, qui pour finir tue le chat si la désintégration a eu lieu (figure I). On est donc dans une situation d'intrication quantique à plusieurs niveaux. Lorsque la probabilité de désintégration est $\mathrm{I} / 2$, le système global atteint une superposition comprenant deux composantes de poids égal, une où le chat est vivant et une où le chat est mort. Schrödinger considère que, de toute évidence, une telle situation est impossible (grotesque) et en conclut, donc, que quelque chose a dû arriver à la chaîne de Von Neumann avant qu'elle n'aille trop loin. À nouveau, la difficulté est d'expliquer l'unicité du monde macroscopique : pourquoi, à un niveau macroscopique, un résultat unique (chat vivant, ou mort) émerge-t-il spontanément, alors que ce n'est pas le cas si l'on se limite strictement à l'équation de Schrödinger.

Le chat est, évidemment, le symbole de n'importe quel objet macroscopique - Einstein, dans une lettre à Schrödinger de la même année [9], prenait l'image de l'explosion macroscopique d'un baril de poudre ${ }^{4}$. Un tel objet ne peut jamais se trouver dans un état « flou »

(4) Einstein écrit « Aucune interprétation, si habile qu'elle soit, ne pourra transformer cette fonction $\Psi$ en une description appropriée des choses réelles; dans la réalité, il n'y a rien entre explosé et non-explosé ». 


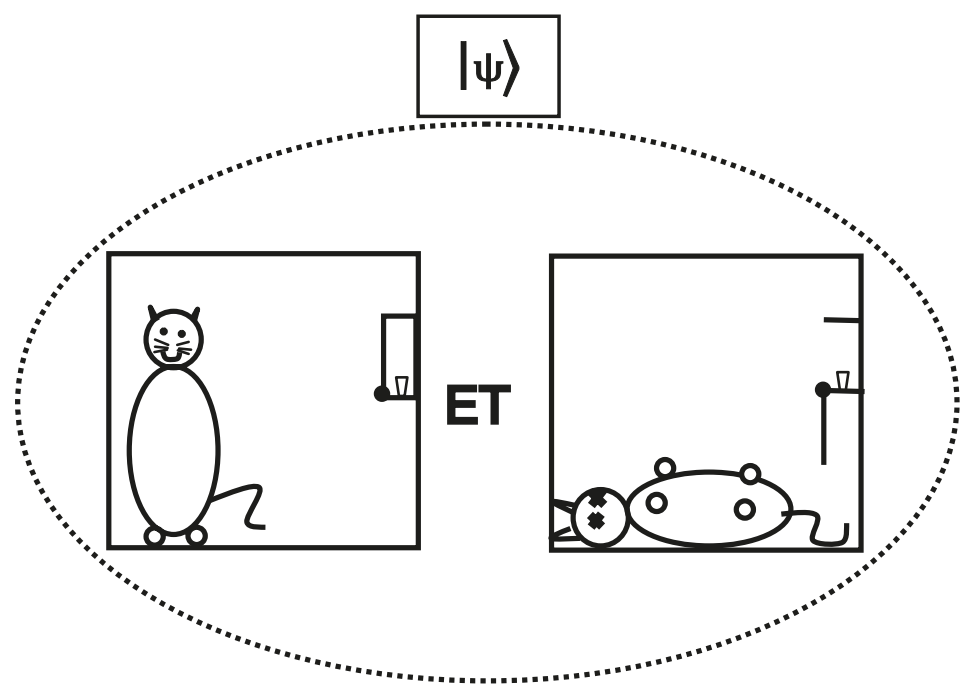

Figure 1. Le paradoxe du chat de Schrödinger illustre comment les règles de la mécanique quantique standard peuvent conduire à des situations extrêmement étranges, où des objets macroscopiques se trouvent en même temps dans des états complètement différents. Schrödinger imagine un chat enfermé dans une boîte qui contient également un dispositif déclenché par l'émission d'une particule par un noyau radioactif. Lorsque la particule est émise, l'appareil se met en marche et ouvre (ou brise) une bouteille de poison, ce qui tue le chat. Si la particule n'est pas émise, le chat reste vivant. Au bout d'un certain temps, lorsque la probabilité d'émission de la particule par le noyau est $1 / 2$, l'état $|\Psi\rangle$ du système total contient des composantes associées à des situations macroscopiques complètement différentes, toutes deux réalisées en même temps. L'unicité de la réalité macroscopique demanderait que le "ET» au milieu de la figure soit remplacé par un "OU», mais ceci n'est pas contenu dans l'équation de Schrödinger. Ce dernier qualifie cette situation de "grotesque ».

contenant des possibilités contradictoires (bouteille ouverte et fermée, chat vivant et mort, etc.). Ce que Schrödinger est donc en train de nous dire est que sa propre équation ne doit pas être poussée trop loin, jusqu'à inclure des objets macroscopiques. La mécanique quantique standard, non seulement est incapable d'éviter de tels cas paradoxaux, mais fournit une recette pour les produire! La conclusion logique est alors qu'un ingrédient supplémentaire est nécessaire pour permettre de sélectionner une seule des branches de la superposition et éviter ces superpositions ridicules ${ }^{5}$. Inutile de dire que la limite de validité de l'équation linéaire n'a pas nécessairement besoin de provenir de l'objet macroscopique lui-même : la sélection d'une branche de la superposition peut par-

(5) L'histoire fait une pirouette amusante en associant le nom de Schrödinger à deux concepts opposés. Le premier est celui d'une équation universelle et puissante, équation continue qui s'applique à tous les systèmes; le second est le chat, le symbole des limites que la même équation ne devrait jamais dépasser. 
faitement avoir lieu bien avant qu'elle n'atteigne le chat. Mais la question vraiment difficile qui subsiste est de savoir où exactement, et quand ce processus de sélection se produit.

L'interprétation standard de la mécanique quantique postule que la seule façon de stopper la propagation linéaire de l'équation de Schrödinger et de casser la régression est de procéder à un acte de mesure. La question devient alors : un animal évolué, ou un être vivant très primitif (bactérie), possède-t-il les capacités intellectuelles suffisantes pour effectuer une mesure et résoudre plusieurs branches de Von Neumann en une seule ? Où se situe la limite entre les êtres vivants capables ou non de percevoir leur propre état et de se projeter eux-mêmes sur un état vivant ou mort ? Ou alors serait-ce que les seuls humains ont accès à un niveau suffisant d'introspection pour devenir conscients de leurs propres observations, et réduire le vecteur d'état ? C'est le point de vue de certaines théories, qui alors impliquent qu'un chat peut effectivement rester dans une superposition d'états vivant et mort pendant très longtemps.

La dernière phrase de la citation de Schrödinger donnée plus haut est souvent considérée comme obscure. II est probable que Schrödinger désire insister sur la différence entre une connaissance incomplète (par une photographie floue) d'un objet bien défini, et un objet qui intrinsèquement n'a pas de frontières bien définies dans l'espace (un nuage) - entre une indétermination qui tient à un manque de connaissance ou une qui est intrinsèque. En d'autres termes, lui aussi remet en question le caractère complet de la mécanique quantique.

\subsection{Malentendus}

Un malentendu classique consiste à penser que le paradoxe du chat est résolu par la simple évocation de la décohérence, qui explique pourquoi il est en pratique totalement impossible d'observer des interférences quantiques entre des états où un chat est vivant ou mort. En fait, la (dé)cohérence n’a rien à voir avec l'argument de Schrödinger : le chat est en réalité un symbole de l'absurdité d'un état quantique qui comprend deux possibilités incompatibles dans la vie courante, que ce soit de façon cohérente ou pas. Cela ne change rien à l'étrangeté de la situation finale que l'état en question soit un état pur (effectivement sujet à décohérence) ou un mélange statistique (qui y est insensible). D'ailleurs, le chat est déjà lui-même part de l'environnement de l'atome radioactif, ainsi que le détecteur et la bouteille de poison ${ }^{6}$. La chaîne (l'arbre des possibilités) commence à se propager à un niveau microscopique (à partir du noyau radioactif) et se propage de plus en plus loin sans limite apparente ; la difficulté est de l'empêcher d'atteindre le monde macroscopique. Cela n'ajoute donc rien de faire remarquer que la chaîne continue au-delà du chat, le mal est déjà fait. Pour finir, invoquer la décohérence n'est pas répondre à la question, c'est juste la répéter.

(6) Le chat lui-même n'est jamais dans une superposition cohérente de vivant ou mort. En effet, lors de la création de la curieuse superposition, le chat est déjà corrélé quantiquement avec la source radioactive, le système mécanique, et la bouteille de poison ouverte ou fermée, le gaz dans la boîte, etc. Toutes ces composantes agissent déjà comme un environnement et produisent une complète décohérence. Rétablir cette cohérence demanderait de mettre toutes ces composantes dans le même état quantique, une tâche clairement impossible. La propagation de la décohérence encore plus loin dans l'environnement n'ajoute rien de nouveau à l'argument. 
La vraie question que pose Schrödinger est comment comprendre l'unicité du monde macroscopique. Comment ce qui était indéterminé peut-il devenir déterminé, et par quel processus ? À quel moment et dans quelles conditions exactes la théorie cesse-t-elle d'être déterministe pour devenir stochastique ? La question qu'il pose n'est donc pas triviale, et il ne suffit pas d'invoquer la décohérence pour y répondre ${ }^{7}$. Schrödinger était d'ailleurs bien au courant des propriétés de l'intrication en mécanique quantique, un mot qu'il a d'ailleurs introduit le premier dans ce contexte (il l'utilise explicitement dans l'article du chat) ; il n'était certainement pas assez naiff pour croire que des chats vivants et morts peuvent interférer. D’ailleurs, dans son article [7] il ne mentionne nulle part le caractère cohérent ou incohérent de la superposition ${ }^{8}$ : pour lui, la question n'est pas d'éviter de se trouver en présence d'une superposition d'états macroscopiques différents, elle est de ne pas avoir de superposition du tout ${ }^{9}$ !

\subsection{Les chats nouvelle vague}

II arrive toutefois que le sens des mots change en physique. Dans la littérature récente en électronique et optique quantiques, il est devenu de plus en plus fréquent d'utiliser les mots « chat de Schrödinger », ou « Schrödinger cat (SC) » dans un sens assez différent, voire presque contradictoire avec le sens initial. Au départ, le chat était en effet le symbole d'une impossibilité, un animal qui ne pourra évidemment jamais exister (une gargouille de Schrödinger ?), l'étape finale d'un raisonnement par l'absurde - bref, un symbole de quelque chose qui n'a jamais existé et n'existera jamais. De nos jours, les mêmes mots sont souvent utilisés pour désigner des états qui sont parfaitement réalisables, à savoir toute superposition cohérente d'états qui sont plus ou moins différents à un niveau le plus macroscopique possible (ici la cohérence est essentielle). Avec cette nouvelle définition, l'existence de chats de Schrödinger a été prévue et effectivement observée pour toute une catégorie de systèmes, par exemple un ion qui se trouve localisé à la fois dans deux positions différentes dans un piège; de tels systèmes subissent évidemment une décohérence rapide du fait de l'apparition de corrélations avec l'environnement. Des calculs théoriques de cette décohérence sont possibles à l'aide de l'équation de Schrödinger, qui peut être utilisée pour calculer comment se déroulent les premières étapes de la chaîne de Von Neumann, et avec quelle rapidité le vecteur d'état tend à se ramifier en plusieurs branches mettant en jeu l'état quantique de l'environnement.

Pour résumer ce $\S 2$, le paradoxe met en lumière la composante centrale de la plupart de nos difficultés avec la mécanique quantique ; comme l'écrit Wigner [10], « les mesures qui laissent le système objet-plus-appareil de mesure dans un état où le pointeur de l'appareil est dans une position bien définie ne peuvent être obtenus dans le cadre des lois linéaires de la mécanique quantique ». La question est alors : quel est donc le processus

(7) L'impossibilité d'observer des interférences entre des états à chat mort et chat vivant est bien évidemment une condition nécessaire pour l'unicité macroscopique. Elle est cependant loin d'être suffisante.

(8) Les mots n'apparaissent pas une seule fois dans l'article.

(9) C'est par exemple le but principal des théories mettant en jeu une dynamique de Schrödinger modifiée, souvent non-linéaire : elles introduisent des équations du mouvement où, lorsque le niveau macroscopique est atteint, toutes les probabilités vont vers zéro du fait de la dynamique, sauf une. 
exact qui force la Nature à rompre cette linéarité et à faire un choix parmi les différents résultats possibles? Il est clair que l'émergence d'un seul résultat est une question essentielle. Comme Pearle le résume de façon concise [II], le problème est d'expliquer « pourquoi les événements se produisent »!

\section{L'ami de Wigner}

Dans une théorie comme la mécanique quantique où l'observateur joue un rôle aussi essentiel, à qui ce rôle peut-il appartenir ? Wigner discute le rôle d'un ami, à qui l'on a demandé d'effectuer une expérience, par exemple une expérience de Stern et Gerlach [12]. L'ami en question travaille à l'intérieur d'un laboratoire totalement fermé, de sorte qu'un observateur extérieur ne peut pas prendre connaissance des résultats de l'expérience avant d'ouvrir la porte du laboratoire (figure 2). Mais quelle est alors la situation juste après que la particule est sortie de l'analyseur et que sa position a été observée dans le laboratoire, tandis qu'elle reste inconnue à l'extérieur ? Depuis dehors, il est naturel de considérer l'ensemble du laboratoire fermé contenant l'expérience et l'ami comme une partie du « système » qui doit être décrit par un grand vecteur d'état. Tant que la porte du laboratoire reste fermée et que le résultat de la mesure est inconnu à l'extérieur, ce vecteur d'état continuera à contenir une superposition des deux résultats; ce n'est que plus tard, lorsque le résultat est connu, qu'il semble pertinent d'appliquer le postulat de réduction du vecteur d'état. Mais, à l'évidence, pour l'ami de Wigner qui est dans le laboratoire, ce raisonnement est tout simplement absurde! Que peut faire l'ouverture de la porte de son point de vue ? II va évidemment préférer considérer que le vecteur d'état est réduit dès que le résultat est observé dans son propre laboratoire. Nous retombons alors sur un point que nous avons déjà discuté, le caractère absolu ou relatif du vecteur d'état : est-ce que cette contradiction signifie que nous devrions prendre en compte deux vecteurs d'état, l'un réduit et l'autre non réduit, pendant la période intermédiaire de l'expérience $^{10}$ ? Pour une discussion par Wigner lui-même du problème de la mesure, voir [10].

Une interprétation souvent associée au nom de Wigner" suppose que la réduction du vecteur d'état est un effet réel qui se produit au moment où un esprit humain acquiert une certaine connaissance du monde physique qui l'entoure, et avec lequel il interagit ; nous y reviendrons dans la suite. On peut par exemple considérer que les courants électriques d'un cerveau humain sont à l'origine de la réduction de l'état quantique des objets mesurés, via un processus physique encore inconnu. Alors, si l'on adopte ce point de vue, la réduction se produit sous l'effet de l'expérimentateur qui se trouve dans le laboratoire (l'ami de Wigner) et la contradiction du paragraphe précédent est levée. Mais, même si on accepte l'idée un peu provocatrice d'une action possible de l'esprit (ou de la conscience) sur l'environnement, ce point de vue ne supprime pas toutes les difficultés logiques:

(I0) Hartle considère que la réponse à cette question est « oui » [13] ; voir également l'interprétation relationnelle (Chapitre $\mathrm{X}$ ).

(II) Le titre de la référence [12] suggère effectivement cette catégorie d'interprétation; de plus, Wigner y écrit que « il s'ensuit (de l'argument de l'ami de Wigner) que la description quantique des objets est influencée par des impressions pénétrant la conscience ». À la fin de l'article, il discute également l'influence de non-linéarités qui pourraient imposer une limite à la validité de l'équation de Schrödinger, et être caractéristiques de la vie. 


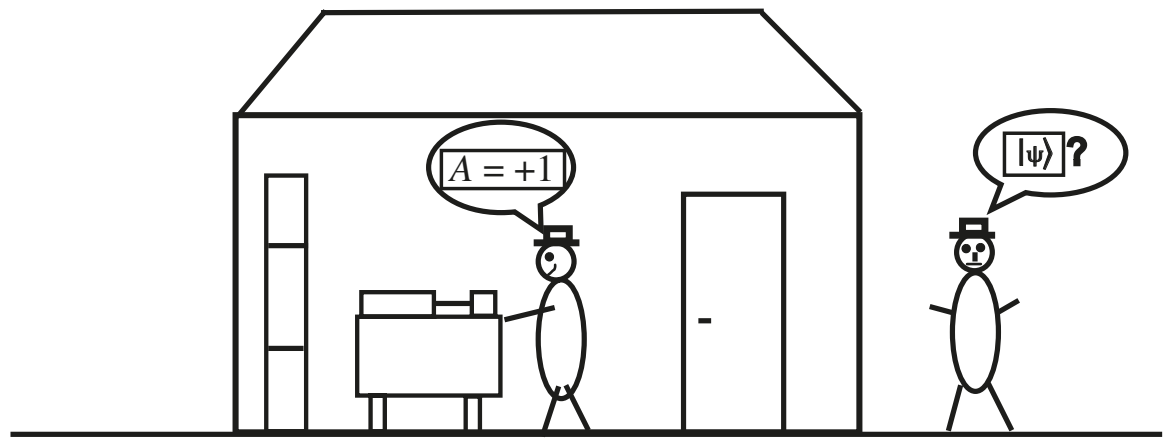

Figure 2. Wigner suppose qu'un physicien à l'extérieur d'un laboratoire a un ami à l'intérieur qui effectue une mesure quantique donnant comme résultats possibles $A= \pm 1$. Lorsque l'ami observe un résultat, par exemple $A=+1$, il décrit le système physique par un vecteur d'état qui inclut cette information, et qui est donc réduit. Cependant, tant que la porte du laboratoire n'est pas ouverte, le physicien dehors décrit le système physique ainsi que son ami par un vecteur d'état qui contient toujours les divers résultats possibles; pour lui, la réduction ne se produit qu'au moment où il ouvre la porte pour prendre connaissance du résultat. On arrive ainsi à une situation où la même réalité physique est décrite par deux vecteurs d'état différents. Pour éviter ce problème, on peut supposer que c'est la première prise de conscience du résultat (celle de l'ami dans le cas présent) qui déclenche la réduction; effectivement le système n'a alors plus qu'un seul vecteur d'état, même si pendant un certain temps il reste inconnu de l'observateur extérieur.
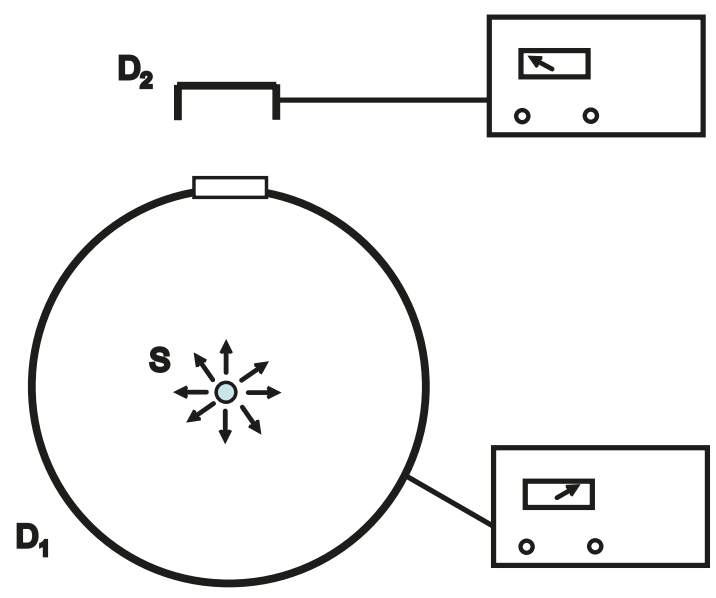

Figure 3. Une source S émet une particule, et se trouve entourée par un détecteur $D_{1}$. Ce dernier capture la particule et enregistre un signal de détection dans un premier appareil de mesure, sauf si la particule passe par un trou correspondant à un petit angle solide (vers le haut sur la figure). Dans ce cas la particule est détectée par $\mathrm{D}_{2}$ et enregistrée par un second appareil. Nous supposons dans la discussion que les détecteurs sont idéaux avec une efficacité de $100 \%$. 
qu'est-ce qu'un esprit humain, quel niveau de prise de conscience est nécessaire pour réduire l'état, comment agissent les courants électriques correspondants, etc. ?

\section{Mesures négatives et " sans interaction »}

D'autres situations paradoxales se produisent en mécanique quantique avec les « mesures négatives », ou « mesures sans interaction ». Ces concepts peuvent être illustrés par les exemples suivants :

(i) Considérons une source qui émet une particule avec une fonction d'onde sphérique (qui prend des valeurs indépendantes de la direction dans l'espace, et ne dépendent donc que de la distance à la source). La source est entourée par deux détecteurs, comme le montre la figure 3 ; un des détecteurs, $\mathrm{D}_{1}$, capture les particules émises dans presque toutes les directions, excepté dans un petit angle solide $\Omega$; le second détecteur $\mathrm{D}_{2}$ capture la particule dans cet angle solide, mais à une plus grande distance. Pour simplifier, nous supposons que l'expérience est idéale et que les détecteurs ont une efficacité de $100 \%$.

Que se passe-t-il maintenant lorsque le paquet d'ondes de la fonction d'onde atteint le premier détecteur? Deux cas sont possibles :

- ou bien la particule est effectivement détectée par D1. Elle disparaît alors, et le vecteur d'état est projeté sur un état sans particule mais avec un détecteur excité (le premier) ; plus tard, le second détecteur $\mathrm{D}_{2}$ ne peut alors plus jamais détecter une particule. Ce premier cas est le plus fréquent.

- ou bien la particule n'est pas détectée. La probabilité d'un tel événement est faible, puisqu'elle est égale au rapport entre l'angle solide sous-tendu par le trou dans le premier détecteur, vu depuis la source, divisé par $4 \pi$ (nous supposons la source isotrope), mais néanmoins elle n'est pas nulle. Alors le simple fait que le premier détecteur n'a pas enregistré la particule entraîne une réduction de la fonction d'onde à sa composante contenue dans l'angle solide $\Omega$, ce qui veut dire que le second détecteur va toujours détecter la particule plus tard. Nous sommes alors en face d'une situation fort curieuse où la probabilité de détection par le second détecteur est fortement augmentée par une sorte de «non-événement » qui s'est produit sur le premier, c'est-à-dire sans aucune interaction entre la particule et l'appareil de mesure. Ceci illustre que la nature profonde du processus de mesure est plus subtile que la "perturbation inévitable des appareils de mesure » tant de fois invoquée (microscope de Heisenberg, etc.) ; en réalité, la réduction du paquet d’ondes peut se produire même lorsque l'interaction avec le premier appareil de mesure ne joue aucun rôle.

Bien sûr, si l'on suppose qu'initialement la particule émise avait une direction d'émission bien définie, alors l'interprétation de cette expérience de pensée devient toute simple : le détecteur 2 n'enregistre que la fraction de particules qui se trouvent émises depuis le début dans sa direction. II n'y a alors rien de surprenant à ce qu'aucune interaction ne soit produite avec le détecteur 1 ! Mais la mécanique quantique orthodoxe postule que cette 
direction d'émission bien définie n'existe pas tant qu'aucune mesure n'a été effectuée ; supposer qu'elle existe revient à ajouter des variables supplémentaires, mais qui n'existe pas dans le cadre de l'interprétation standard de la mécanique quantique.

(ii) La figure 4 montre une expérience semblable : une source $S$ émet des particules une par une, chacune décrite par une onde de Schrödinger se propageant vers la droite. Cette onde interagit avec un dispositif de type lame séparatrice qui la scinde en deux parties (les photons, les neutrons, et d'autres particules peuvent ainsi être séparés avec des dispositifs appropriés). Après s'être propagée sur une distance $\mathrm{L}_{1}$ ou $\mathrm{L}_{2}$, chacune des ondes tombe sur un détecteur, $\mathrm{D}_{1}$ dans un cas, $\mathrm{D}_{2}$ dans l'autre. Lors de chaque réalisation de l'expérience, une seule particule est émise par $S$, et une seule particule est détectée, soit par $\mathrm{D}_{1}$ soit par $\mathrm{D}_{2}$. Dans la description de l'expérience purement en termes d'onde de Schrödinger, la situation reste parfaitement symétrique : en chacun des détecteurs, une chaîne de von Neumann intervient, mettant en jeu des états des détecteurs où la particule a été ou n'a pas été enregistrée. Mais la mécanique quantique prédit qu'un seul résultat de mesure est observé : pour une réalisation donnée, soit la particule est détectée en $D_{1}$, soit en $D_{2}$, mais jamais aux deux endroits. $S i L_{1}<L_{2}$, la première mesure est celle en $D_{1}$, et il en résulte un effet de mesure négative semblable au précédent : si $D_{1}$ ne détecte pas la particule, elle est toujours détectée en $\mathrm{D}_{2}$. Inversement, lorsqu'elle est détectée en $\mathrm{D}_{1}$, il devient certain qu'elle ne sera jamais détectée en $\mathrm{D}_{2}$. Voilà qui donne déjà un avantgoût de la non-localité quantique : une détection en $\mathrm{D}_{1}$ rend immédiatement impossible une détection en $\mathrm{D}_{2}$, même si la distance entre les détecteurs est arbitrairement grande (de sorte qu'aucune information se propageant à la vitesse de la lumière n'a le temps de se propager de l'un à l'autre). La résolution de la chaîne de von Neumann sur l'un des détecteurs (réduction du vecteur d'état si l'on préfère) est un phénomène non-local dont les conséquences se font immédiatement sentir sur des mesures situées à des distances arbitrairement grandes.

Comme plus haut, on peut remarquer que les mêmes résultats sont facilement explicables par un modèle local : il est suffisant de supposer que, lors de chaque réalisation de l'expérience, la particule « choisit » au hasard une seule direction lorsqu'elle croise la lame séparatrice. La difficulté semble alors ne venir que de la description inhabituelle des phénomènes donnée par la mécanique quantique. On peut montrer qu'il existe des exemples de cas où aucun modèle local ne peut être inventé pour reproduire les prédictions quantiques.

(iii) Considérons maintenant le dispositif schématisé sur la figure 5, avec un interféromètre de Mach-Zhender dans lequel une source émet une par une des particules, qui sont ensuite comptées sur les détecteurs $D_{1}$ et $D_{2}$. Comme le fait la référence [14], nous supposons que les différences de chemins sont réglées de façon à créer une interférence destructive dans la voie de sortie du détecteur $\mathrm{D}_{2}$; aucune particule ne peut alors atteindre ce détecteur de sorte que toutes sont détectées en $\mathrm{D}_{1}$ (à nouveau, nous supposons les détecteurs parfaits avec un rendement de $100 \%$ ). Que se produit-il maintenant si l'on insère un objet opaque $\mathrm{O}$ dans le bras inférieur de l'interféromètre ? On annule l'effet d'interférence destructive, ce qui permet à certaines particules d'atteindre $\mathrm{D}_{2}$; cet événement se produit une fois sur 4 si les deux lames semi-réfléchissantes ont des transmissions de $50 \%$. Dans un tel cas, comme la particule n'a pas été bloquée par l'objet, il semble que 


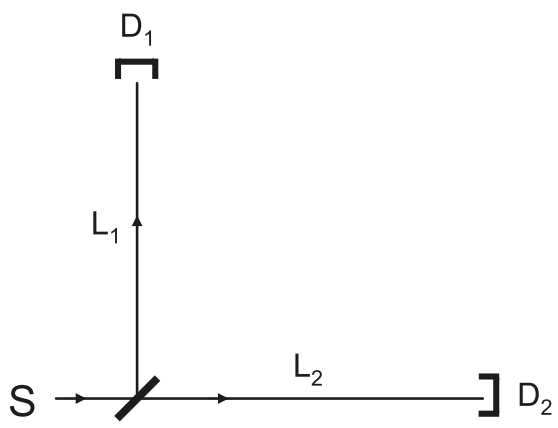

Figure 4. Des particules sont émises une par une par une source S. Les ondes de Schrödinger décrivant les particules sont scindées en deux par une lame séparatrice; chaque composante se propage vers un détecteur, $D_{1}$ ou $D_{2}$. Dans une expérience idéale, lors de chaque réalisation la particule est toujours détectée, soit en $D_{1}$ soit en $D_{2}$ et de façon totalement aléatoire ; la particule n'est jamais détectée aux deux endroits.

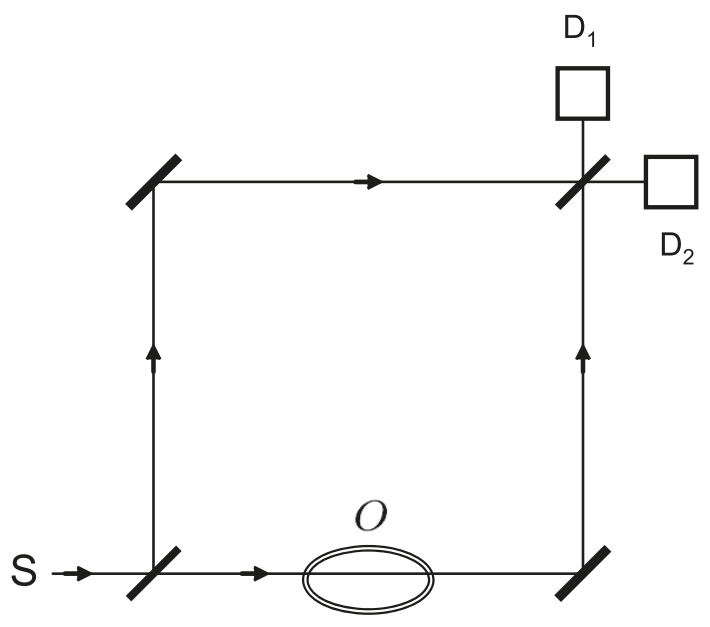

Figure 5. Une source S émet une série de particules, une par une, vers un interféromètre (Mach-Zehnder). La différence de chemin de ce dernier est ajustée de façon que toutes les particules atteignent le détecteur $D_{1}$ et qu'aucune particule ne puisse atteindre $D_{2}$. Lorsqu'un objet opaque O est inséré afin de bloquer un des chemins pour la particule, l'effet d'interférence destructive ne se produit plus et des particules sont parfois détectées en $D_{2}$. Dans un tel événement, la présence d'un objet est mesurée avec certitude, alors qu'il semble qu'aucune interaction avec l'objet n'est mise en jeu, puisque la particule détectée est nécessairement passée par le chemin supérieur dans l'interféromètre (sinon elle aurait été absorbée). 
c'est nécessairement qu'elle est passée par le bras du haut de l'interféromètre, ce qui veut dire qu'elle n'a jamais rencontré l'objet et pu être absorbée par lui. Mais, inversement, si l'objet n'avait pas été inséré, jamais la particule n'aurait pu atteindre $\mathrm{D}_{2}$ ! Le résultat final est que la seule observation d'une détection en $\mathrm{D}_{2}$ révèle la présence d'un objet, bien que le processus exclue toute interaction avec cet objet. Ce phénomène est appelé « mesure sans interaction ${\aleph^{\prime 2}}^{2}$. II illustre bien que, tant que la localisation d'une particule n'a pas été mesurée, il est vain en mécanique quantique standard de lui attribuer une position ou une trajectoire unique ; c'est en quelque sorte le mesure positive en $\mathrm{D}_{2}$ qui « annule » à distance les effets de l'interaction entre la particule et l'objet opaque $\mathrm{O}$, qui ressort totalement inchangé de cette réalisation particulière de l'expérience ${ }^{13}$.

Pour des raffinements de ces idées et la discussion d'expériences reliées aux mesures sans interaction, voir $[15,16]$. Hardy a proposé une version mettant en jeu un double interféromètre, un pour un électron et un pour un positron, et l'annihilation mutuelle de ces particules [17].

Une application possible est une méthode appelée « imagerie sans interaction » [I8], où des images d'objets peuvent être obtenues sans les perturber. Elle pourrait être utilisée à la réalisation d'une technique non-invasive de microscopie électronique, en particulier pour la visualisation de molécules biologiques sans risque de les détruire [20]. Il a également été suggéré que les mesures négatives puissent se révéler utiles dans le contexte de la cryptographie quantique. L'idée proposée dans [2I] est que deux partenaires éloignés, Alice et Bob, effectuent aléatoirement des choix entre deux polarisations orthogonales ; Alice envoie un photon avec la polarisation qu'elle a choisie à Bob, qui renvoie le photon à Alice grâce à un miroir si, et seulement si, sa polarisation diffère du choix local qu'il a effectué. L'ensemble du dispositif comprend un interféromètre qui est réglé de façon que, si le photon revient de Bob vers Alice, la probabilité qu'Alice puisse observer un photon sur un détecteur $D_{1}$ s'annule, à cause d'un effet d'interférence destructive tout à fait semblable à celui de l'exemple (ii) ci-dessus. Dans ces conditions, en sélectionnant seulement les événements où Alice observe un photon en $\mathrm{D}_{1}$ (de sorte qu'alors Bob ne peut détecter la particule), on sélectionne automatiquement des événements où les deux choix aléatoires faits par Alice et Bob se sont trouvés être identiques. Si Alice et Bob se communiquent mutuellement le résultat de chaque expérience (quel détecteur a cliqué ou non), mais conservent secrets leurs choix de polarisations, en faisant une liste de leurs choix binaires locaux pour les événements sélectionnés, ils se constituent progressivement une clé secrète. Une caractéristique remarquable de ce dispositif est que les événements pris en compte pour la transmission secrète de la clé sont en fait ceux où aucun photon n’a été

(I2) Même si le phénomène demande que la particule test puisse interagir avec l'objet : il est clair que l'effet ne se produirait pas si l'objet était totalement transparent pour la particule. On peut résumer l'événement en disant que l'objet a absorbé l'onde associée à la particule, mais pas la particule elle-même.

(13) En d'autres termes, le processus de mesure sélectionne effectivement une composante du vecteur d'état qui s'est propagée dans le bras supérieur de l'interféromètre seulement. Cependant, cet événement n'est rendu possible que par le fait que, pendant sa propagation dans le bras inférieur, l'onde de Schrödinger a été absorbée. La chose curieuse est que la particule et son onde semblent dissociées dans ce processus, puisqu'elles se propagent dans des bras différents, situés à une distance arbitrairement grande l'un de l'autre. 
transmis, puisqu'il est resté à l'intérieur de l'appareillage utilisé par Alice ${ }^{14}$ : on est donc dans une situation analogue à l'exemple (i) ci-dessus (puisque la détection de la particule signifie qu'elle n'a pas pris le chemin contenant l'objet). Du fait que la particule ne suit pas la ligne de transmission entre les deux sites dans les événements pris en compte pour la construction de la clé secrète, le flux de particules sur cette ligne ne contient absolument aucune information concernant les choix aléatoires de polarisations faits par Alice et Bob - d'où une excellente confidentialité.

\section{Une grande variété de points de vue}

Les citations qui suivent peuvent être utiles pour se faire une idé $e^{15}$ de la variété des positions intéressantes qui ont été exprimées depuis l'apparition de la mécanique quantique.

\section{Interprétation de Copenhague :}

(i) Bohr ([19], 2e édition, page 204 et [22]) : « II n'existe pas de monde quantique. II n'existe qu'une description physique abstraite. II est faux de penser que la tâche de la physique est de découvrir comment la Nature est réellement. Ce qui concerne la physique est ce que nous pouvons dire sur la Nature ». Ou, de façon similaire : « II n'existe pas de concept quantique » [23]. Pour ce qui est des phénomènes physiques : « on peut plaider vigoureusement pour une limitation de l'usage du mot phénomène au cas où il se réfère exclusivement à des observations, obtenues dans des circonstances bien spécifiées, incluant une description de l'ensemble de l'expérience » [24].

Bohr définit également l'objet de la physique de la façon suivante $[25,26]$ : « La physique doit être considérée, non pas tellement comme l'étude de quelque chose qui est fourni a priori, mais plutôt comme le développement de méthodes qui permettent de classer et de rendre compte de l'expérience humaine. De ce point de vue, notre tâche doit être de rendre compte de cette expérience d'une façon qui soit indépendante de jugements individuels subjectifs, qui donc est objective au sens qu'elle peut être communiquée de façon non-ambigüe dans un langage humain ordinaire $»$.

Alors que la mécanique quantique est souvent considérée (y compris de nos jours) comme une théorie complètement nouvelle par rapport à la physique classique (une révolution), Bohr préfère la voir comme son extension logique naturelle. II considère [27, 28] qu'à l'introduction de la mécanique quantique «le problème auquel les physiciens se sont trouvés confrontés était de développer une généralisation rationnelle de la physique

(14) Les événements utiles sont ceux où Bob n'a pas renvoyé vers Alice l'onde de Schrödinger, mais sans absorber la particule. Chaque fois qu'Alice et Bob choisissent la même polarisation, le vecteur d'état comprend une composante où la particule se propage vers le site de Bob, de sorte qu'on pourrait mesurer sa présence le long de la ligne de transmission ou sur le site de Bob. Mais cette composante s'annule au moment où Alice observe la particule en $\mathrm{D}_{1}$.

(15) Avec, bien sûr, la précaution habituelle : il est vrai que de courtes citations peuvent, lorsqu'elles sont isolées de leur contexte, donner une idée un peu superficielle de la position précise de leurs auteurs. 
classique ${ }^{16}$ permettant d'incorporer de façon harmonieuse le concept de quantum d'action ». Dans ce but, l'outil principal est le « principe de correspondance » [29] : " Le principe de correspondance exprime la tendance à utiliser dans un développement systématique de la théorie quantique chaque caractéristique des théories classiques, en une transcription rationnelle qui soit appropriée au contraste fondamental entre les postulats des théories classiques et quantiques ». II est donc naturel qu'il ait mis l'accent, à de nombreuses reprises, sur l'importance des concepts classiques pour donner une signification au formalisme de la mécanique quantique ; en particulier, les concepts classiques sont indispensables lorsqu'il faut décrire un processus de mesure.

Bohr a écrit de nombreux textes sur la mécanique quantique, parfois assez tournés vers la philosophie [30,31] ; nous proposerons plus loin d'autres de ses citations, dont certaines illustrent les vues de Bohr sur l'espace-temps. Dans sa fameuse conférence de Côme de septembre 1927 [32], il introduit la notion de « complémentarité », relation entre attributs contradictoires d'un même objet en physique quantique, qu'ensuite il élargit bien au-delà, allant jusqu'à inclure d'autres disciplines que la physique (voir par exemple [30] ou encore [24] où il mentionne la biologie ${ }^{17}$, la sociologie et la psychologie; voir également le $\S 7.2$ de [19] ou le chapitre 4 de [6]).

(ii) Born en 1926 (page 804 de [33]) : « Le mouvement des particules se conforme aux lois de la probabilité, mais la probabilité elle-même se propage selon les lois de la causalité $»$.

Ou peu après dans [34] : « La description théorique quantique... ne répond pas... à la question de savoir où se trouve une certaine particule à un instant donné... Ceci suggère que la mécanique quantique ne répond qu'aux questions statistiques correctement posées, et ne dit rien de l'évolution de phénomènes individuels. Ce serait alors une fusion singulière entre mécanique et statistique $»$.

(iii) Heisenberg $[19,35]$ : « Mais les atomes ou les particules élémentaires ne sont pas réels ; ils forment un monde de potentialités ou de possibilités, plutôt qu'un monde de choses et de faits $)$.

Dans «Physics and philosophy » [35] (chapitre V) : " Les sciences de la nature ne donnent pas simplement une description et une explication de la nature; elles font partie des interactions entre la nature et nous-mêmes; elles décrivent la nature telle qu'elle réagit à notre méthode pour lui poser des questions ».

Le chapitre III de ce livre a pour titre « L'interprétation de Copenhague de la théorie quantique », et il y écrit : « Nous ne pouvons complètement objectiver le résultat d'une observation, nous ne pouvons décrire ce qui 'se produit' entre cette observation et la suivante ».

(16) De façon semblable, Bohr voyait probablement la relativité d'Einstein comme une généralisation rationnelle de l'électromagnétisme classique (équations de Maxwell).

(17) À la fin de cet article, il discute le «mode de description par complémentarité » et illustre sa généralité en écrivant « Un exemple est donné par la biologie où les arguments mécaniques et vitalistes sont utilisés d'une façon typique de la complémentarité. En sociologie également, une telle dialectique peut se révéler souvent utile, en particulier dans les problèmes où nous sommes confrontés avec la comparaison entre les différentes cultures humaines... ». 
Plus bas, il ajoute : « En conséquence, la transition entre le 'possible' et le 'réalisé' se produit pendant l'acte d'observation. Si nous voulons décrire ce qui se produit pendant un événement atomique, nous devons réaliser que le mot 'se produit' ne peut s'appliquer qu'à l'observation, et pas à l'état des choses entre les deux observations ».

II conclut ce chapitre avec : « l'appareil de mesure a été construit par l'observateur, et nous devons nous souvenir que ce que nous observons n'est pas la nature en elle-même mais la nature soumise à notre méthode de questionnement. Notre tâche scientifique en physique consiste à poser des questions concernant la Nature dans le langage que nous possédons, et à essayer d'obtenir une réponse de l'expérience par les moyens qui sont à notre disposition. C'est ainsi que la théorie quantique nous rappelle, comme Bohr l'a dit, la vieille sagesse qui dit que, lorsque nous sommes à la recherche d'une harmonie dans la vie, nous ne devons jamais oublier que nous sommes à la fois acteurs et spectateurs dans le drame de la vie. Il est compréhensible que, dans notre relation scientifique avec la nature, notre propre activité devienne très importante lorsque nous nous préoccupons de parties de la nature auxquelles nous n'avons accès que par les outils les plus élaborés ».

(iv) Jordan (tel que cité par Bell dans [36]) : « les observations ne se contentent pas de perturber ce qui doit être mesuré, elles le produisent. Dans une mesure de la position, l'électron est forcé de prendre une décision. Nous le contraignons à occuper une position bien précise ; auparavant, il n'était ni ici ni là, il n'avait pris aucune décision concernant une position précise... ».

(v) Landau and Lifchitz, au début du premier chapitre de leur livre sur la mécanique quantique [37] : « La possibilité de la description quantitative du mouvement de l'électron exige également l'existence d'êtres physiques obéissant avec une précision suffisante à la mécanique classique. Si un électron entre en interaction avec un 'être classique', alors l'état de ce dernier change en général... Ceci étant, l'être classique est appelé ordinairement appareil, et on parle de son processus d'interaction avec l'électron comme d'une mesure. Il convient toutefois de souligner qu'on n'a alors nullement en vue un processus de mesure où participe un physicien observateur. Par mesure on entend en mécanique quantique tout processus d'interaction d'un être classique et d'un être quantique, se déroulant par ailleurs indépendamment de tout observateur. On doit à N. Bohr d'avoir élucidé le rôle profond de la notion de mesure en mécanique quantique ».

(vi) Dirac, page 7 de [38] : « Le seul objet de la physique théorique est de calculer des résultats qui peuvent être comparés avec l'expérience, et il est tout à fait inutile de donner une description globale de l'ensemble du phénomène ».

\section{Critiques de l'interprétation de Copenhague :}

(vii) Schrödinger : «le monde ne m'est donné qu'une seule fois, et pas une fois pour son existence et une autre pour sa perception. Le sujet et l'objet sont un. La barrière qui les sépare ne peut être considérée comme brisée du seul fait d'une expérience en sciences physiques, pour la simple raison que cette barrière n'existe pas » [39]. Dans le §2, nous donnons d'autres citations de Schrödinger. 
(viii) Einstein, dans une lettre de 1928 à Schrödinger [40, 4I] : « La philosophie rassurante de Heisenberg - ou est-ce une religion ? - est construite de façon tellement subtile que, pour le moment, elle fournit un oreiller de paresse pour le véritable croyant, oreiller d'où il n'est pas possible de le réveiller ».

En 1936 [42] : « La fonction $\Psi$ ne décrit en aucune manière une condition qui pourrait concerner un seul système ; elle concerne en fait un grand nombre d'entre eux, un 'ensemble de systèmes' au sens de la mécanique statistique... si la fonction $\Psi$ ne fournit que des données statistiques sur les grandeurs mesurées... la raison en tient ... au fait que la fonction $\Psi$ ne décrit d'aucune manière l'état du système unique $»$.

(ix) de Broglie [43] : « L'interprétation de la Mécanique ondulatoire de Bohr et Heisenberg a de nombreuses conséquences qui ouvrent des perspectives philosophiques nouvelles. Le corpuscule n'est plus un objet bien défini dans le cadre de l'espace et du temps ; il n'est plus qu'un ensemble de potentialités affectées de probabilités, il n'est plus qu'une entité qui se manifeste à nous de façon fugitive, tantôt sous un aspect, tantôt sous un autre. M. Bohr, qui est un peu le Rembrandt de la Physique contemporaine, car il manifeste parfois un certain goût pour le 'clair-obscur', a dit des corpuscules qu'ils sont 'unsharply defined indidivuals within finite space-time limits' ».

\section{Plus récemment :}

(x) Bell [2], lorsqu'il décrit la théorie quantique « moderne » (interprétation de Copenhague) et ses relations avec la cosmologie : « cette théorie ne parle jamais d'événements se produisant dans le système, mais seulement des résultats d'observations faites sur lui, ce qui implique l'existence d'un appareillage externe $\rangle^{18}$ (si c'est le cas, comment alors décrire l'Univers entier, puisqu'il est impossible de concevoir un appareillage externe dans ce cas ?). « Le problème est le suivant : la mécanique quantique ne s'intéresse qu'aux observations. Elle divise donc nécessairement le monde en deux, une partie qui est observée et une autre qui effectue l'observation. Les résultats dépendent de la façon dont est faite cette division, mais aucune règle précise pour le faire n'est proposée. Tout ce dont nous disposons est une recette qui, du fait des limitations pratiques auxquelles sont sujets les humains, est suffisamment non-ambigüe pour toute question pratique ».

Voir aussi son texte « contre le concept de mesure », où il discute et critique diverses présentations de l'interprétation orthodoxe [44].

(xi) Mermin [45], lorsqu'il résume la « doctrine quantique fondamentale » (interprétation orthodoxe) : « le résultat d'une mesure n'accède à l'existence que par la mesure elle-même, étant une conséquence à la fois de l'état du système mesuré et de l'appareil de mesure. Comment exactement ce résultat précis est obtenu dans une mesure particulière - la transition entre la potentialité et le réel selon Heisenberg - est fondamentalement impossible à savoir $»$.

(I8) On pourrait ajouter « ainsi que des observateurs externes ». 
(xii) Shimony [46] : " D'après l'interprétation proposée par Bohr, le changement de l'état est une conséquence de l'hypothèse fondamentale selon laquelle la description de tout phénomène physique demande de faire référence au dispositif expérimental ».

(xiii) Rosenfeld [47], parlant de l'interprétation orthodoxe : « l'observateur humain, que nous avions pris tant de soin à faire sortir du paysage (scientifique), semble s'introduire à nouveau de façon irrésistible,... ».

(xiv) Gottfried [48] (page 188 de la première édition) : « Le postulat de réduction est un axiome indépendant... Le résultat de ces considérations est que la mécanique quantique ne peut pas donner une description complète du monde physique car il doit exister des systèmes (appelés « conscients » par Wigner) qui sortent du domaine d'application de la théorie, c'est-à-dire qui ne peuvent pas être incorporés dans la partie du monde que nous décrivons par l'équation de Schrödinger ».

(xv) Stapp [49] : « L'interprétation de la théorie quantique est assombrie par les points suivants : (I) On attribue à des concepts classiques non-valides un statut fondamental ; (2) Le processus de la mesure ne peut pas être décrit dans le cadre de la théorie ; (3) La distinction sujet-objet devient floue ; (4) Le système observé doit d'une part être isolé pour être défini, mais d'autre part interagir pour être observé »

Ou, cité par Bell dans [2] : « Comment une théorie qui est fondamentalement une procédure par laquelle des créatures macroscopiques grossières, telles que des êtres humains, calculent des probabilités concernant ce qu'ils vont observer dans des circonstances macroscopiques, comment cette théorie pourrait-elle être considérée comme une description complète de la réalité ? »

(xvi) Leggett [50] à propos de l'interprétation de Copenhague : « ... le formalisme de la mécanique quantique connu sous le nom d'interprétation de Copenhague (alors qu'il serait probablement plus correct de l'appeler la non-interprétation de Copenhague, du fait qu'elle implique que toute tentative d'interpréter le formalisme est voué à l'échec)... d'une part nie que des objets microscopiques (électrons, photons, atomes,...) aient nécessairement des propriétés définies en l'absence d'observation, mais d'autre part stipule avec insistance (ou du moins implique) que des objets macroscopiques (compteurs, chats, etc.) possèdent effectivement de telles propriétés, qu'ils soient observés ou non. Cette insistance sur la nécessité de tracer une ligne nette... entre le monde microscopique et le monde macroscopique de la vie de tous les jours (les appareils de mesure inclus) est un thème constamment présent dans les écrits de Niels Bohr... ». Dans [5I], il commente : «La difficulté réelle commence seulement quand nous prenons au sérieux le fait que l'appareil de mesure... est lui-même un système constitué d'atomes et d'électrons, de sorte qu'en principe il devrait pouvoir être décrit dans les termes de la mécanique quantique. II devrait donc être légitime de demander ce qui se produit si, au lieu de traiter la mesure comme quelque chose de complètement étranger au comportement habituel des systèmes physiques, nous la traitons tout simplement comme un type particulier de processus physique et la décrivons par l'équation de Schrödinger linéaire dépendant du temps ». 
Puis, dans [52], il commente : « Dans le cadre de l'interprétation conventionnelle de la mécanique quantique, un système ne possède pas de propriétés bien définies jusqu'au moment où, en quelque sorte, nous le forçons à nous indiquer ces propriétés par la réalisation de mesures appropriées. Mais est-ce la seule interprétation possible ?... à un niveau plus profond il est parfaitement possible que les systèmes aient, en fait, des propriétés objectives, que quelqu'un effectue des mesures sur lui ou non... Les résultats apparemment aléatoires prédits par le formalisme quantique seraient alors simplement dûs à notre ignorance des détails à un niveau plus profond de description ».

(xvii) Van Kampen [53] a émis le caveat amusant suivant : « Toute personne qui attribuera au vecteur d'état plus de signification que ce qui est nécessaire pour calculer des phénomènes observables sera tenue responsable des conséquences! „N Notons deux autres références $[54,55]$ qui mettent en pratique cet avertissement.

\section{La situation actuelle :}

Comme on peut s'en douter à la lecture de ces citations, loin d'être convergentes ou parfois même contradictoires, aucun consensus général n'a réellement émergé au sein de toute la communauté des physiciens concernant le sens précis du vecteur d'état. L'accord est général en ce qui concerne le formalisme et la façon d'utiliser en pratique le vecteur d'état. Pour toutes les expériences qui ont été réalisées, un choix pragmatique entre les deux postulats d'évolution peut être laissé à l'évaluation du physicien ; pour faire des prédictions concrètes sur une expérience, jusqu'à maintenant un peu de bon sens s'est révélé suffisant, de sorte que les problèmes liés aux fondements de la théorie quantique peuvent parfaitement être mis de côté dans les laboratoires. Mais ceci n'empêche pas qu'il serait préférable de disposer de préceptes mathématiques précis, au lieu de devoir se contenter de recettes physiquement raisonnables ! II n'est donc pas surprenant de constater que, lorsque la question des fondements revient sur le tapis, ou celle du sens précis des objets mathématiques de la mécanique quantique, le débat recommence, et parfois devient passionné. De plus, on constate que même ceux qui annoncent être en total accord avec l'interprétation standard font usage, dans la pratique, de toute une série de nuances (pouvant parfois aller jusqu'à la contradiction interne...) dès qu'on leur demande d'expliquer en détail leur point de vue.

Pour résumer, le statut du vecteur d'état en mécanique quantique orthodoxe est un mélange subtil entre différents concepts concernant la réalité et la connaissance que nous pouvons avoir de cette réalité. Les fantastiques succès de la théorie quantique standard signifient-ils que le vecteur d'état est réellement la description ultime et la plus précise d'un système quantique que la physique ne donnera jamais dans les siècles à venir ? Faut-il renoncer à toute description réaliste en physique et adopter un point de vue positiviste ? La question n'est pas réglée. II faut dire que même Bohr est considéré plus comme un réaliste que comme un positiviste ou un opérationnaliste [19]. Comme le dit Jammer ([6], p I57) : « Bohr, comme Von Weizsäcker [56] l'a souligné, n’a jamais rejeté la notion de réalité, il l'a seulement modifiée ». Si on lui avait demandé quelles sont exactement les relations entre la fonction d'onde et la réalité, Bohr aurait probablement dit que la fonction d'onde est assurément un outil très utile, mais que le concept de réalité ne peut 
être défini à ce seul niveau de façon correcte ; il doit inclure tous les appareils de mesure macroscopiques qui servent à avoir accès à l'information microscopique.

\section{Des arguments peu convaincants}

Nous avons déjà souligné combien l'invention de l'interprétation standard (ou de Copenhague) de la mécanique quantique a été, et reste, un des grands triomphes de la physique. On ne peut qu'admirer, plus encore avec le recul, la rapidité et la perspicacité avec laquelle les fondateurs du domaine l'ont conçue, à une époque où les données expérimentales étaient aussi peu nombreuses. Mais, depuis, de multiples expériences ont été réalisées, dont une partie destinées précisément à trouver les limites de cette interprétation ou à la mettre en défaut ; pas une n'y a réussi pour le moment. C'est donc une sorte de miracle de l'analyse et de la logique qui a permis l'émergence de cette fantastique construction intellectuelle.

Cela dit, il faut bien admettre que, parfois, les remarquables auteurs de cette construction sont allés trop loin, poussés par leur désir de convaincre. Par exemple, des affirmations définitives ont été proférées concernant la nécessité logique absolue d'adopter l'interprétation standard, affirmations qui paraissent maintenant quelque peu exagérées avec le recul du temps. D'après elles, l'interprétation orthodoxe donnerait la seule et unique description ultime possible de la réalité physique ; jamais une description plus fine ne serait possible. Ainsi, le caractère fondamentalement indéterministe des phénomènes microscopiques devrait être considéré comme une loi de la Nature gravée dans le marbre pour l'éternité. Mais nous savons maintenant que ce n'est pas nécessairement la seule possibilité logique ; oui bien sûr, chacun est libre de préférer l'interprétation standard, mais ce n'est pas la seule possible à partir d'arguments purement scientifiques. D'autres interprétations restent parfaitement possibles, le déterminisme en lui-même n'étant pas du tout éliminé de façon définitive ${ }^{19}$. Comme expliqué dans la référence [45] par exemple, et comme Bell l'a vu clairement très tôt $[57,58]$, les « preuves d'impossibilité » mises en avant pas les défenseurs de l'interprétation de Copenhague ne sont pas rigoureuses, pour une raison toute simple : elles imposent arbitrairement des conditions qui sont peut-être pertinentes au sein de la mécanique quantique (linéarité), mais n'ont aucune raison de l'être dans le cadre des théories à rejeter - n'importe quelle théorie à variables supplémentaires comme celle de Bohm par exemple. Du fait de la stature scientifique exceptionnelle des auteurs de ces preuves d'impossibilité, la communauté des physiciens a mis longtemps avant de réaliser que ces théorèmes étaient non pertinents ; maintenant que ce fait est reconnu, la pluralité des interprétations de la mécanique quantique est acceptée plus facilement.

(19) Pourvu que l'on accepte la non-localité. 


\section{Références}

[I] J.S. Bell, Speakable and Unspeakable in Quantum Mechanics, Cambridge University Press (1987) ; seconde édition complétée (2004), qui contient la série complète des articles de J. Bell sur la mécanique quantique.

[2] J.S. Bell, Quantum mechanics for cosmologists, in Quantum Gravity, C. Isham, R. Penrose, and D. Sciama eds., 2, 611-637, Clarendon Press (1981) ; pages 117-138 de [1].

[3] N.D. Mermin, Is the moon there when nobody looks? Reality and the quantum theory, Phys. Today 38, 38-47 (April 1985).

[4] F. London et E. Bauer, La théorie de l'observation en mécanique quantique, $n^{\circ} 775$ des Actualités scientifiques et industrielles, exposés de physique générale ; Hermann, Paris (1939); traduit en anglais dans The theory of observation in quantum mechanics in Quantum Theory of Measurement, J.A. Wheeler and W.H. Zurek eds., Princeton University Press, pp. 217-259 (1983); voir en particulier $\$ 1$, mais aussi 13 et 14.

[5] J. von Neumann, Mathematische Grundlagen der Quantenmechanik, Springer, Berlin (1932) ; Mathematical Foundations of Quantum Mechanics, Princeton University Press (1955).

[6] M. Jammer, The Philosophy of Quantum Mechanics, Wiley (1974).

[7] E. Schrödinger, Die gegenwärtige Situation in der Quantenmechanik, Naturwissenschaften 23, 807-812, 823-828, 844-849 (1935).

[8] J.D. Trimmer, The present situation in quantum mechanics: a translation of Schrödinger's cat paradox paper, Proc. Amer. Phil. Soc. 124, 323-338 (1980). Also available in pp. 152-167, Quantum Theory of Measurement, J.A. Wheeler and W.H. Zurek eds, Princeton University Press (1983).

[9] A. Einstein, lettre à Schrödinger du 8 août 1935, reproduite par exemple page 238 de $[4 I]$.

[10] E.P. Wigner, The problem of measurement, Am. J. Phys. 31, 6-15 (1963) ; reproduit dans Symmetries and Reflections, Indiana University Press, pp. 153-170; ou encore dans Quantum Theory of Measurement, J.A. Wheeler and W.H. Zurek eds., Princeton University Press (1983), pp. 324-341.

[II] P. Pearle, Toward explaining why events occur, Int. J. Theor. Phys. 18, 489-518 (1979).

[12] E.P. Wigner, Remarks on the mind-body question in The scientist speculates, I.J. Good ed., Heinemann, London (196I), pp. 284-302 ; reproduit dans E.P. Wigner, Symmetries and Reflections, Indiana University Press (1967), pp. 17|-184.

[13] J.B. Hartle, Quantum mechanics of individual systems, Am. J. Phys. 36, 704-712 (1968).

[14] A.C. Elitzur et L. Vaidman, Quantum mechanical interaction-free measurements, Found. Phys. 23, 987-997 (1993).

[15] P. Kwiat, H. Weinfurter, T. Herzog, A. Zeilinger, et M.A. Kasevich, Interaction-free measurement, Phys. Rev. Lett. 74, 4763-4766 (1995).

[16] L. Hardy, On the existence of empty waves in quantum theory, Phys. Lett. A 167, II-16 (1992). 
[17] L. Hardy, Quantum mechanics, local realistic theories, and Lorentz invariant realistic theories, Phys. Rev. Lett. 68, 298I-2984 (1992).

[18] A.G. White, J.R. Mitchell, O. Nairz et P. Kwiat, Interaction-free imaging, Phys. Rev. A 58, 605-613 (1998).

[19] M. Jammer, The Conceptual Development of Quantum Mechanics, Mc Graw Hill (1966), deuxième édition (1989).

[20] W. Putnam et M. Yanik, Noninvasive electron microscopy with interaction-free quantum measurements, Phys. Rev. A 80, 040902 (2009).

[21] Tae-Gon Noh, Counterfactual quantum cryptography, Phys. Rev. Lett. 103, 23050 I (2009).

[22] A. Petersen, The philosophy of Niels Bohr, in Bulletin of the Atomic Scientists XIX, 8-14 (September 1963).

[23] C. Chevalley, Niels Bohr's words and the Atlantis of Kantianism, in Niels Bohr and Contemporary Philosophy, J. Faye and H. Folse eds., Dordrecht Kluwer (1994), pp. 33-57.

[24] N. Bohr, On the notions of causality and complementarity, Dialectica 2, 312-319 (1948).

[25] N. Bohr, The unity of human knowledge (October 1960) ; Atomic Physics and Human Knowledge, Wiley (1958 et 1963).

[26] C. Norris, Quantum Theory and the Flight from Realism: Philosophical Responses to Quantum Mechanics, Routledge (2000), p. 233.

[27] N. Bohr, Quantum physics and philosophy: causality and complementarity, in Philosophy in the Mid-Century: A Survey; R. Klibansky ed., La Nuova Italia Editrice, Firenze (1958). Voir également The quantum of action and the description of nature, in Atomic Theory and the Description of Nature, Cambridge University Press (1934), pp. 92-101.

[28] P. Bokulich et A. Bokulich, Niels Bohr's generalization of classical mechanics, Found. Phys. 35, 347-37I (2005).

[29] N. Bohr, Atomic theory and mechanics, Nature II6, 845-852 (1925).

[30] N. Bohr, Collected Works, edited by F. Aaserud, Elsevier (2008); voir également Collected Works, Complementarity beyond Physics (1928- 1962).

[31] N. Bohr, Atomic Physics and Human Knowledge, Wiley (1958), et Dover (201I), voir en particulier « Discussions with Einstein on epistemological problems in atomic physics 》 ou, avec une bibliographie et un glossaire: C. Chevalley: Physique atomique et connaissance humaine, folio essais, Gallimard (1991) ; Essays 1933 to 1957 on Atomic Physics and Human Knowledge, Ox Bow Press (1987) ; Essays 1958-62 on Atomic Physics and Human Knowledge, Wiley (1963) et Ox Bow Press (1987) ; Atomic Physics and the Description of Nature, Cambridge University Press (1934 et 1961).

[32] N. Bohr, The quantum postulate and the recent development of atomic theory, Nature, Supplement April 14, 1928, 580-590 (1928).

[33] M. Born, Quantenmechanik der Stossvorgänge, Zeitschrift für Physik 38, 803-827 (1926) ; Zur Wellenmechanik der Stossvorgänge, Göttingen Nachrichten 146-160 (1926). 
[34] M. Born, Physical aspects of quantum mechanics, Nature I19, 354- 357 (1927).

[35] W. Heisenberg, Physics and Philosophy, Harper \& Brothers (1958); Harper Perennial Modern Classics (2007).

[36] J.S. Bell, Bertlmann's socks and the nature of reality, J. Physique colloques C 2, 4I-62 (198I). Cet article est reproduit dans les pages 139-158 de [1].

[37] L.D. Landau et E.M. Lifshitz, Quantum Mechanics, Non-Relativistic Theory, Pergamon Press (1958), Butterworth-Heinemann Ltd (1996).

[38] P.A.M. Dirac, The Principles of Quantum Mechanics, Oxford University Press (1930, 1958).

[39] E. Schrödinger, What is Life? Mind and Matter, Cambridge University Press (1944 et 1967), p. 137.

[40] A. Einstein, lettre à Schrödinger du 31 mai 1928, reproduite par exemple page 213 de $[4 \mid]$.

[4I] F. Balibar, O. Darrigol, et B. Jech, Albert Einstein, oeuvres choisies I, quanta, Editions du Seuil et Editions du CNRS (1989).

[42] A. Einstein, Physik und Realität, Journal of the Franklin Institute 221, 313-347 (1936).

[43] L. de Broglie, La physique quantique restera-t-elle indéterministe?, Revue des sciences et de leurs applications, 5, 289-31I (1952). Académie des Sciences, session du 25 avril 1953, http://www.sofrphilo.fr/telecharger.php?id=74

[44] J.S. Bell, Against measurement, in Sixty Two Years of Uncertainty: Historical, Philosophical and Physical Enquiries into the Foundations of Quantum Mechanics, Erice meeting in August 1989, A.I. Miller ed. (Plenum Press); reproduit dans pp. 213-23I de l'édition 2004 de [I].

[45] N.D. Mermin, Hidden variables and the two theorems of John Bell, Rev. Mod. Phys. 65, 803-8I5 (1993); voir en particulier le §IIII.

[46] A. Shimony, Role of the observer in quantum theory, Am. J. Phys. 3I, 755-773 (1963).

[47] L. Rosenfeld, The measuring process in quantum mechanics, Suppl. Prog. Theor. Phys., extra number 222 Commemoration of the thirtieth anniversary of the meson theory by Dr. H. Yukawa (1965).

[48] K. Gottfried, Quantum Mechanics, Benjamin (1966) ; deuxième édition, K. Gottfried et Yan Tun-Mow, Springer (2003).

[49] H.P. Stapp, S-matrix interpretation of quantum theory, Phys. Rev. D 3, 1303-1320 (197I).

[50] A.J. Leggett, Testing the limits of quantum mechanics: motivation, state of play, prospects, J. Phys. Condens. Matter I4, R4I5-R45I (2002).

[5I] A.J. Leggett, Macroscopic quantum systems and the quantum theory of measurement, Supplement of the Progr. Theor. Phys. n 69, 80- 100 (1980).

[52] A.J. Leggett, The Problems of Physics, Oxford University Press (1987).

[53] N.G. van Kampen, Ten theorems about quantum mechanical measurements, Physica A I53, 97-113 (1988). 
[54] B.G. Englert, M.O. Scully, et H. Walther, Quantum erasure in double-slit interferometers with which-way detectors, Am. J. Phys. 67, 325-329 (1999); voir les premières lignes du §IV.

[55] C.A. Fuchs et A. Peres, Quantum theory needs no 'interpretation', Phys. Today 53, March 2000, 70-71 ; voir également diverses réactions à ce texte dans les lettres publiées dans le numéro de septembre 2000.

[56] C.F. von Weizsäcker, Voraussetzungen des naturwissenschaftlichen Denkens, Hanser Verlag (197I) et Herder (1972).

[57] D. Bohm et J. Bub, A proposed solution of the measurement problem in quantum mechanics by a hidden variable theory, Rev. Mod. Phys. 38, 453-469 (1966).

[58] D. Bohm et J. Bub, A refutation of the proof by Jauch and Piron that hidden variables can be excluded in quantum mechanics, Rev. Mod. Phys. 38, 470-475 (1966). 
